\title{
DIAGNÓSTICO DE CÂNCER DE MAMA: PERFIL SOCIOECONÔMICO, CLÍNICO, REPRODUTIVO E COMPORTAMENTAL DE MULHERES
}

Franciéle Marabotti Costa Leite ${ }^{1}$, Cintya Raquel Araújo Gonçalves ${ }^{2}$, Maria Helena Costa Amorim³, Susana Bubach ${ }^{4}$

\begin{abstract}
RESUMO: Estudo descritivo, com abordagem quantitativa, cujo objetivo foi traçar o perfil socioeconômico, clínico, comportamental e reprodutivo de mulheres do Município de São Mateus - Espírito Santo que vivenciaram o diagnóstico de câncer de mama no período de 2005 a 2010. Foram entrevistadas 23 mulheres, de janeiro a abril de 2011. Aproximadamente $70 \%$ estavam na faixa etária de 36 a 49 anos e $\geq 60$ anos; $60,9 \%$ eram casadas ou viviam em união consensual; 47,8\% possuíam o ensino fundamental incompleto e 39,2\% pertenciam à classe D; 26,1\% possuíam histórico familiar de neoplasia mamária e 78,2\% descobriram o câncer através do autoexame; $52,2 \%$ foram submetidas à cirurgia do tipo não conservadora; $87 \%$ já engravidaram e $60 \%$ tiveram a primeira gestação entre 20 a 29 anos; $78,3 \%$ realizavam o autoexame e $87 \%$ a mamografia. O estudo permitiu identificar demandas e comportamentos passíveis de mudanças.
\end{abstract}

PALAVRAS-CHAVE: Neoplasias da mama; Saúde da mulher; Enfermagem.

\section{DIAGNOSIS OF BREAST CANCER: SOCIO-ECONOMIC, CLINICAL, REPRODUCTIVE AND BEHAVIORAL PROFILE OF THE WOMEN}

\begin{abstract}
A descriptive study with a quantitative approach, whose objective was to outline the socio-economic, clinical, behavioral and reproductive profile of the women of the town of São Mateus - in the state of Espírito Santo who were living through the diagnosis of breast cancer in the period from 2005 to 2010. 23 women were interviewed, between January and April 2011. Approximately $70 \%$ were in the age range 36 to 49 and $\geq 60 ; 60,9 \%$ were married or cohabiting; $47.8 \%$ had not finished their primary education and $39,2 \%$ belonged to class D; $26,1 \%$ had a family history of mammary neoplasm and $78,2 \%$ had discovered the cancer through self-examination; $52,2 \%$ had undergone non-conservative surgery; $87 \%$ had already been pregnant and $60 \%$ had their first pregnancy between 20 and 29 years of age; 78,3\% carried out self-examinations and $87 \%$ the mammography. The study allowed the identification of needs and behaviors which may be changed.
\end{abstract}

KEYWORDS: Breast neoplasms; Women's health; Nursing.

\section{DIAGNÓSTICO DE CÁNCER DE MAMA: PERFIL SOCIOECONÓMICO, CLÍNICO, REPRODUCTIVO Y DEL COMPORTAMIENTO DE MUJERES}

RESUMEN: Estudio descriptivo, con abordaje cuantitativo, cuyo objetivo fue trazar el perfil socioeconómico, clínico, del comportamiento y reproductivo de mujeres del Municipio de São Mateus - Espírito Santo, que tuvieron el diagnóstico de cáncer de mama en el periodo de 2005 a 2010. Fueron entrevistadas 23 mujeres, de enero a abril de 2011. Aproximadamente $70 \%$ estaban con edad entre 36 y 49 años y $\geq 60$ años; $60,9 \%$ eran casadas o vivían en unión consensual; 47,8\% tenían enseñanza fundamental incompleta y 39,2\% pertenecian a la clase D; 26,1\% poseían histórico familiar de neoplasia mamaria y $78,2 \%$ descubrieron el cáncer a través del autoexamen; $52,2 \%$ fueron sometidas a cirugía del tipo no conservadora; $87 \%$ ya se quedaron embarazadas y $60 \%$ tuvieron la primera gestación entre 20 y 29 años; 78,3\% realizaban el autoexamen y 87\% la mamografía. El estudio permitió identificar demandas y comportamientos pasibles de cambios.

PALABRAS CLAVE: Neoplasias de mama; Salud de la mujer; Enfermería.

\footnotetext{
${ }^{1}$ Enfermeira. Mestre em Saúde Coletiva. Professora do Departamento de Enfermagem da Universidade Federal do Espírito Santo-UFES. ${ }^{2}$ Acadêmica de Enfermagem da UFES.

${ }^{3}$ Enfermeira. Doutora em Enfermagem. Professora do Departamento de Enfermagem da UFES.

${ }^{4}$ Enfermeira. Mestre em Saúde Coletiva. Professora do Departamento de Ciências da Saúde da UFES.
}

Autor correspondente:

Franciéle Marabotti Costa Leite

Universidade Federal do Espírito Santo

Rua Lumberto Maciel de Azevedo, 405 - 29090-700 -Vitória-ES-Brasil

E-mail: emaildafran@ig.com.br
Recebido: 03/01/2012 Aprovado: 14/03/2012 


\section{INTRODUÇÃO}

O câncer tem alcançado altos índices de prevalência e está entre as principais causas de morte no mundo, o que requer preparação dos profissionais de saúde para atuar nesta realidade ${ }^{(1)}$. No Brasil, dados do Instituto Nacional de Câncer (INCA) revelam, para o ano de 2012, 518.510 novos casos de câncer, dos quais 260.640 na população feminina. Vale destacar que, sem considerar a neoplasia do tipo não melanoma, o tumor mamário é o mais frequente entre as mulheres. Espera-se em 2012, no país, 52.680 novos casos de câncer de mama com incidência de 52 casos para cada 100 mil mulheres, já no Espírito Santo a ocorrência será de 49,42 casos por $100 \mathrm{mil}^{(2)}$.

A neoplasia mamária, quando diagnosticada e tratada oportunamente, possui bom prognóstico, pois diminui a mortalidade e melhora a sobrevida ${ }^{(3)}$. Existem diferentes modalidades terapêuticas que são aplicadas de forma individualizada, sendo a cirurgia (mastectomia, quadrantectomia ou tumorectomia) e a radioterapia tratamentos locais, e a quimioterapia e hormonioterapia, tratamentos sistêmicos ${ }^{(4)}$.

O INCA preconiza a detecção precoce do câncer de mama por meio do Exame Clínico das Mamas (ECM), realizado por um profissional de saúde devidamente treinado, e a mamografia. Apesar do autoexame das mamas (AEM) não ser considerado no rastreamento da neoplasia mamária, este deve ser estimulado nas ações de educação em saúde, a fim de que a mulher possa conhecer o seu corpo, e realizar periodicamente o exame das mamas ${ }^{(4)}$.

A Estratégia de Saúde da Família (ESF) e o Programa de Agentes Comunitários de Saúde (PACS), portas de entrada e da ampliação do acesso aos serviços de saúde, atuam na promoção da saúde, nas atividades de informação e de orientação, assistindo integralmente a população de seu território, a partir das demandas identificadas ${ }^{(5)}$, dessa forma têm papel fundamental não apenas no rastreamento do câncer de mama, mas no seguimento da mulher com diagnóstico desta neoplasia.

Diante do exposto, justifica-se a necessidade da realização de um estudo para se conhecer o perfil da mulher que vivencia o diagnóstico de câncer de mama, a fim de se ter elementos concretos para atuar nas ESF/PACS, bem como, para outros níveis de atenção e que possibilitem reflexões acerca da assistência à essa população na região norte do Estado do Espírito Santo. Nesse contexto, o objetivo deste estudo consiste em traçar o perfil socioeconômico, clínico, comportamental e reprodutivo de mulheres do Município de São Mateus-Espírito Santo, que vivenciaram o diagnóstico de câncer de mama no período de 2005 a 2010.

\section{MÉTODO}

Trata-se de um estudo do tipo descritivo, transversal, com abordagem quantitativa, desenvolvido no período de janeiro a abril de 2011, com 23 mulheres que vivenciaram o diagnóstico de câncer de mama no período de 2005 a 2010, cadastradas nas ESF ou PACS, do Município de São Mateus. Este município possui 106.964 habitantes $^{(6)}$ e cobertura de 78\% de ESF/PACS.

As mulheres foram convidadas a fazer parte da pesquisa e, após a orientação e especificação quanto aos objetivos do estudo e assinatura do Termo de Consentimento Livre e Esclarecido, foi realizada entrevista com registro das informações em dois formulários. $\mathrm{O}$ primeiro para registrar as variáveis sociodemográficas (faixa etária, situação conjugal e escolaridade); variáveis clínicas (história de câncer de mama na família, como descobriu o nódulo na mama, tempo de diagnóstico, tipo de cirurgia realizada e outros tratamentos); variáveis reprodutivas (número de gestações e idade da primeira gestação) e variáveis comportamentais (realização e periodicidade do AEM e mamografia antes e após o diagnóstico). O segundo instrumento foi utilizado para registrar dados de identificação da classe econômica das participantes, por meio da Classificação Econômica Brasil da Associação Brasileira de Empresas de Pesquisa (ABEP), que estima o poder de compra das pessoas e famílias urbanas, abandonando a pretensão de classificar a população em termos de classes sociais ${ }^{(7)}$.

Empregou-se os programas Excel XP e Word XP, para digitação e criação do banco de dados para análise, a qual ocorreu por meio do programa Social Package Statistical Science (SPSS) 15.0. Os resultados foram apresentados na forma descritiva com a utilização de frequência relativa (\%) e absoluta $(\mathrm{N})$. Este estudo foi aprovado pelo Comitê de Ética em Pesquisa (CEP) do Centro Universitário do Norte do Espírito Santo, sob o número de registro 029/2010.

\section{RESULTADOS}

A tabela 1 apresenta os dados socioeconômicos das mulheres, percebe-se que a faixa etária de 36 a 49 anos e 60 anos ou mais obtiveram o mesmo percentual de $34,8 \%$, representando aproximadamente $70 \%$. No que se refere à situação conjugal, $60,9 \%$ eram casadas ou em união consensual; $47,8 \%$ possuíam o ensino fundamental incompleto e $39,2 \%$ das mulheres pertencíam à classe $\mathrm{D}$.

Quanto ao perfil clínico (Tabela 2), 26,1\% possuem histórico de câncer de mama na família; a maioria 
Tabela 1 - Perfil socioeconômico de mulheres com diagnóstico de câncer de mama, de 2005 a 2010. São Mateus, 2011

\begin{tabular}{lcc}
\hline \multicolumn{1}{c}{ Variáveis } & N & \% \\
\hline Faixa etária & 8 & 34,8 \\
36 a 49 anos & 7 & 30,4 \\
50 a 59 anos & 8 & 34,8 \\
60 anos ou mais & & \\
Situação conjugal & 14 & 60,9 \\
Casada / União consensual & 4 & 17,4 \\
Viúva & 3 & 13,0 \\
Solteira & 2 & 8,7 \\
Divorciada & & \\
Escolaridade & 4 & 17,4 \\
Analfabeta & 11 & 47,8 \\
Fundamental incompleto & 0 & 0,0 \\
Fundamental completo & 1 & 4,3 \\
Médio incompleto & 3 & 13,0 \\
Médio completo & 1 & 4,3 \\
Superior incompleto & 3 & 13,0 \\
Superior completo & & \\
Classe econômica & 5 & 21,7 \\
Classe B2 & 2 & 8,7 \\
Classe C1 & 7 & 30,4 \\
Classe C2 & 9 & 39,2 \\
Classe D & 23 & 100,00 \\
Total & &
\end{tabular}

$(78,2 \%)$ referiu ter descoberto o nódulo durante o autoexame das mamas. Observa-se que grande parte $(47,8 \%)$ tem entre um e dois anos de diagnóstico, $52,2 \%$ foram submetidas à cirurgia do tipo não conservadora e aos quatro tipos de tratamento; $61 \%$ das mulheres que vivenciaram o câncer estavam em tratamento hormonal.

A tabela 3 mostra o perfil reprodutivo, observa-se que $87 \%$ das participantes já engravidaram, e que a maioria (60\%) teve de um a três filhos. Quanto à idade da primeira gestação, percebe-se que $60 \%$ tiveram o primeiro filho na faixa etária de 20 a 29 anos.

Os dados das variáveis comportamentais (Tabela 4) evidenciaram que cerca de $70 \%$ das entrevistadas realizavam o AEM antes do diagnóstico, a maioria $(62,5 \%)$ o fazia eventualmente. Após o diagnóstico, constatou-se que $78,3 \%$ das mulheres afirmaram realizar o AEM, entretanto, $72,2 \%$ o realizam mensalmente.

A realização da mamografia antes do diagnóstico de câncer de mama era feita por cerca de $61 \%$, e a maioria $(78,6 \%)$, realizava anualmente. Após o diagnóstico $87 \%$ das mulheres se submetem a mamografia, e $60 \%$ a realiza semestralmente.
Tabela 2 - Perfil clínico de mulheres com diagnóstico de câncer de mama, de 2005 a 2010. São Mateus, 2011

\begin{tabular}{|c|c|c|}
\hline Variáveis & $\mathbf{N}$ & $\%$ \\
\hline \multicolumn{3}{|l|}{ História de câncer na família } \\
\hline $\operatorname{Sim}$ & 6 & 26,1 \\
\hline Não & 17 & 73,9 \\
\hline \multicolumn{3}{|c|}{ Como descobriu o nódulo na mama } \\
\hline Autoexame & 18 & 78,2 \\
\hline Mamografia & 4 & 17,4 \\
\hline Ultrassonografia & 1 & 4,3 \\
\hline \multicolumn{3}{|l|}{ Tempo de diagnóstico } \\
\hline Menos de 1 ano & 5 & 21,7 \\
\hline Mais de 1 e menos de 2 anos & 11 & 47,8 \\
\hline Entre 2 e menos de 3 anos & 3 & 13,0 \\
\hline Entre 3 e 5 anos & 4 & 17,4 \\
\hline \multicolumn{3}{|l|}{ Tipo de Cirurgia } \\
\hline Conservadora & 11 & 47,8 \\
\hline Não conservadora & 12 & 52,2 \\
\hline \multicolumn{3}{|l|}{ Tratamentos realizados } \\
\hline $\mathrm{Cx}, \mathrm{Rt}, \mathrm{Qt}$ & 5 & 21,7 \\
\hline $\mathrm{Cx}, \mathrm{Rt}, \mathrm{Ht}$ & 1 & 4,3 \\
\hline $\mathrm{Cx}, \mathrm{Rt}, \mathrm{Qt}, \mathrm{Ht}$ & 12 & 52,2 \\
\hline $\mathrm{Cx}, \mathrm{Qt}$ & 4 & 17,4 \\
\hline $\mathrm{Cx}, \mathrm{Ht}$ & 1 & 4,3 \\
\hline \multicolumn{3}{|l|}{ Hormonioterapia atual } \\
\hline $\operatorname{Sim}$ & 16 & 60,9 \\
\hline Não & 7 & 39,1 \\
\hline Total & 23 & 100,00 \\
\hline
\end{tabular}

Legenda: Cx - Cirurgia; Rt - Radioterapia;

Qt - Quimioterapia; Ht - Hormonoterapia

Tabela 3 - Perfil reprodutivo de mulheres que vivenciaram o diagnóstico de câncer de mama no período de 2005 a 2010. São Mateus, 2011

\begin{tabular}{lcc}
\hline \multicolumn{1}{c}{ Variáveis } & N & \% \\
\hline Já engravidou? & & \\
$\quad$ Sim & 20 & 87,0 \\
Não & 3 & 13,0 \\
Número de gestações & 12 & 60,0 \\
$1-3$ & 8 & 40,0 \\
$\geq 4$ & 5 & 25,0 \\
Idade em que teve a primeira gestação & \\
15 a 18 anos & 12 & 60,0 \\
20 a 29 anos & 3 & 15,0 \\
30 a 38 anos & 23 & 100,00 \\
Total & & \\
\hline
\end{tabular}


Tabela 4 - Perfil comportamental de mulheres que vivenciaram o diagnóstico de câncer de mama no período de 2005 a 2010. São Mateus, 2011

\begin{tabular}{|c|c|c|}
\hline Variáveis & $\mathbf{N}$ & $\%$ \\
\hline \multicolumn{3}{|c|}{$\begin{array}{l}\text { Realizava o autoexame antes do diagnóstico de } \\
\text { câncer de mama }\end{array}$} \\
\hline Sim & 16 & 69,6 \\
\hline Não & 7 & 30,4 \\
\hline \multicolumn{3}{|l|}{ Periodicidade? } \\
\hline Realizava eventualmente & 10 & 62,5 \\
\hline Realizava mensalmente & 6 & 37,5 \\
\hline Total & 16 & 100,0 \\
\hline \multicolumn{3}{|c|}{ Atualmente realiza o autoexame de mama } \\
\hline Sim & 18 & 78,3 \\
\hline Não & 5 & 21,7 \\
\hline \multicolumn{3}{|l|}{ Periodicidade? } \\
\hline Realizava eventualmente & 5 & 27,8 \\
\hline Realizava mensalmente & 13 & 72,2 \\
\hline Total & 18 & 100,0 \\
\hline \multicolumn{3}{|c|}{$\begin{array}{l}\text { Realizava a mamografia antes do diagnóstico de } \\
\text { câncer de mama }\end{array}$} \\
\hline Sim & 14 & 60,9 \\
\hline Não & 9 & 39,1 \\
\hline \multicolumn{3}{|l|}{ Periodicidade? } \\
\hline Anualmente & 11 & 78,6 \\
\hline Mais de 3 anos & 3 & 21,4 \\
\hline Total & 14 & 100,0 \\
\hline \multicolumn{3}{|c|}{ Atualmente realiza mamografia } \\
\hline $\operatorname{Sim}$ & 20 & 87,0 \\
\hline Não & 3 & 13,0 \\
\hline \multicolumn{3}{|l|}{ Periodicidade? } \\
\hline De 6 em 6 meses & 12 & 60,0 \\
\hline Anualmente & 7 & 35,0 \\
\hline Não respondeu & 1 & 5,0 \\
\hline Total & 20 & 100,0 \\
\hline Total & 23 & 100,0 \\
\hline
\end{tabular}

\section{DISCUSSÃO}

A maior ocorrência de participantes na faixa etária de 36 a 49 anos e 60 anos ou mais é semelhante ao estudo de coorte histórica realizado com população feminina com câncer de mama. A faixa etária de 50 a 59 anos representou $28,8 \%$ das mulheres em estudo realizado $^{(8)}$. Quanto à maioria ser casada ou em união consensual, possuir o ensino fundamental incompleto e pertencer à classe $\mathrm{D}$, esses resultados coadunam com outra pesquisa realizada ${ }^{(9)}$.

A história familiar constitui um importante fator de risco para o desenvolvimento do câncer de mama ${ }^{(4)}$.
O presente estudo revela que $26,1 \%$ das mulheres afirmaram ter histórico de neoplasia mamária na família. Pesquisa destaca que cerca de $22 \%$ das pacientes tinham história familiar de tumor de mamário ${ }^{(10)}$.

Observa-se no presente estudo, que a maioria das participantes afirma ter descoberto o nódulo na mama por meio do AEM, achado que vai ao encontro dos dados apresentados pelo Ministério da Saúde ${ }^{(11)}$ que revela que cerca de $80 \%$ dos tumores são descobertos pela própria mulher.

Percebe-se que a maior parcela foi submetida à cirurgia do tipo não conservadora, dado que vai ao encontro de estudo que aponta a realização de mastectomia, como tipo de cirurgia predominante $(53 \%)^{(12)}$. A utilização cada vez maior da cirurgia do tipo conservadora, em que não há extirpação completa da mama, tem reduzido o efeito negativo na autoimagem e imagem corporal das mulheres afetadas pela doença ${ }^{(13)}$.

A maioria das participantes do estudo foi submetida aos quatro tipos de tratamento (cirurgia, quimioterapia, radioterapia e hormonioterapia). Em estudo desenvolvido sobre a assistência oncológica pelo Sistema Único de Saúde, em mulheres com câncer de mama no Estado do Rio de Janeiro, verificou-se que a maioria delas que realizou quimioterapia foi submetida a tratamento local (cirurgia e radioterapia) e a tratamento sistêmico (hormonioterapia) ${ }^{(14)}$.

A hormonioterapia vem sendo utilizada em pacientes com receptores de estrogênio e/ou receptores de progesterona positivos, e vem sendo recomendada por aumentar a sobrevida de pacientes com câncer de mama $^{(14)}$. No presente estudo percebe-se que a maioria das mulheres estava em tratamento hormonal. Pesquisa revela que $73,8 \%$ das pacientes tratadas com câncer de mama em Centro de Alta Complexidade em Oncologia e $61 \%$ das mulheres assistidas nas Unidades Isoladas, estavam em hormonioterapia ${ }^{(14)}$.

No que tange à nuliparidade como fator de risco para o desenvolvimento da neoplasia mamária ${ }^{(15)}$, notase que grande parte das entrevistadas já engravidou, e a idade da primeira gestação foi na faixa etária de 20 a 29 anos. Esse achado corrobora com os dados de outro estudo que mostra que a maior parte das mulheres com diagnóstico de câncer de mama teve a primeira gestação na faixa etária de 21 a $30 \operatorname{anos}^{(16)}$.

A evidência de que grande parte das entrevistadas realizava o AEM antes do diagnóstico, é apresentado por este estudo. Pesquisa com mulheres no município de Botucatu-SP identificou que 78,9\% realizavam o autoexame $^{(17)}$. Quando questionadas sobre a periodicidade, $62,5 \%$ afirmaram realizar eventualmente. Esse achado se 
contrapõe ao de estudoque aponta a realização eventual do AEM por aproximadamente $43 \%$ das mulheres ${ }^{(18)}$. Quanto à realização deste exame após o diagnóstico do tumor mamário, constata-se o aumento no percentual de mulheres que passaram a realizá-lo, assim como a periodicidade de sua realização. Esse exame, apesar de ser uma prática passível, não é recomendado pelo Ministério da Saúde, que enfatiza como principais estratégias de rastreamento populacional a mamografia e o ECM. Entretanto, deve ser estimulado nas ações de educação em saúde, a fim de que a população possa conhecer o seu corpo ${ }^{(4)}$.

A realização da mamografia antes do diagnóstico de câncer de mama era feita por cerca de $61 \%$, percentual superior aos achados em estudo desenvolvido nas unidades de saúde da família do Município de AracruzEspírito Santo, o qual demonstrou que aproximadamente $56 \%$ das mulheres já tinham realizado mamografia ${ }^{(19)}$. No presente estudo, da população que já havia sido submetida à mamografia, $78,6 \%$ a realizava anualmente. $\mathrm{O}$ Ministério da Saúde enfatiza o rastreamento do câncer de mama, por meio da mamografia, pelo menos a cada dois anos para mulheres de 50 a 69 anos, ou no caso de grupos populacionais considerados de risco elevado para neoplasia mamária (com história familiar de câncer de mama em parentes de primeiro grau), recomenda-se a mamografia anualmente, a partir de $35 \operatorname{anos}^{(4)}$.

Após o diagnóstico o percentual de mulheres que passou a ser submetida a mamografia aumentou, e a maioria passou a realizá-la a cada seis meses. Outro dado interessante é a existência de mulheres que afirmam não terem feito este exame após o diagnóstico. $\mathrm{O}$ documento de Consenso do Controle do Câncer de mama recomenda como seguimento para mulher com câncer de mama a realização do exame mamográfico a cada 12 meses $^{(4)}$. Vale lembrar ainda, que além deste procedimento, o ECM é considerado um exame de rastreamento do tumor mamário e deve ser feito em todas as mulheres que procuram o serviço de saúde, independentemente da faixa etária, como parte do atendimento, devendo, no caso de mulheres com neoplasia mamária, ser feito semestralmente nos primeiros cinco anos de diagnóstico e anualmente após este período ${ }^{(4)}$.

\section{CONCLUSÃO}

Conclui-se que a maioria das mulheres do Município de São Mateus-ES que vivenciaram o diagnóstico de câncer de mama e são assistidas pela ESF ou PACS, encontra-se na faixa etária de 36 a 49 anos e 60 anos ou mais, possuem o ensino fundamental incompleto, pertencem à classe $\mathrm{D}$, tem histórico familiar de neopla- sia mamária e descobriram o tumor por meio do AEM. Observa-se a prevalência da realização de cirurgia do tipo não conservadora e dos quatro tipos de tratamento (cirurgia, quimioterapia, radioterapia e hormonioterapia). Nota-se que grande parte já engravidou e a idade da primeira gestação foi na faixa etária de 20 a 29 anos.

A maioria realizava o AEM antes do diagnóstico, entretanto após o diagnóstico houve um aumento na adesão a este exame. Apesar de o autoexame ser importante para o processo de conhecimento do corpo, este não pode ser visto como forma de rastreamento do câncer de mama, assim como não deve substituir o ECM, que deve ser feito por um profissional de saúde devidamente treinado. Dessa forma, considerando que neste estudo não foi avaliado a forma como o AEM é realizado, a ESF e o PACS devem estar atentos a esta prática, avaliar a realização por parte da população e prover oficinas e atividades de educação e informação em saúde.

Outro ponto a destacar foi o aumento do número de mulheres que passaram a realizar a mamografia com periodicidade semestral, bem como número de mulheres que após o diagnóstico ainda não realizaram este exame. Sugere-se investigar o grupo que ainda não realizou a mamografia após o diagnóstico e o que está realizando acima do que recomenda o Consenso do Controle do Câncer de Mama. Em tempo, a ESF e o PACS devem criar estratégias que garantam o acesso aos serviços de saúde, estimulando a prevenção secundária da neoplasia mamária por meio da oferta do exame mamográfico e do ECM por um profissional devidamente treinado.

O conhecimento do perfil de mulheres com câncer de mama permite identificar demandas e comportamentos passíveis de mudanças, e juntamente com o apoio da ESF e PACS, pode oportunizar um seguimento adequado a esta população, bem como ampliar a oferta da prevenção primária e secundária.

\section{AGRADECIMENTOS}

À Fundação de Amparo a Pesquisa do Estado do Espírito Santo(FAPES), pelo apoio à realização da pesquisa, conforme processo número: 51206188-10.

\section{REFERÊNCIAS}

1. Kalinke LP, Kochla KRA, Labronici LM, Lima T, Visentin A, Testoni R. Evolução das pacientes submetidas à cirurgia de mama em drenagem aspirativa. Cogitare enferm. 2011;16(4):689-94. 
2. Brasil. Ministério da Saúde. Instituto Nacional de Câncer. Estimativa 2012: incidência de câncer no Brasil. Rio de Janeiro, 2011 [acesso em 25 de dez 2011]. Disponível: http://www.inca.gov.br/estimativa/2012/ estimativa20122111.pdf

3. Albrecht CAM. Análise de sobrevida de pacientes com câncer de mama atendidas no Hospital Santa Rita de Cássia, na cidade de Vitória, Espírito Santo. [dissertação]. Vitória(ES): Universidade Federal do Espírito Santo; 2011.

4. Brasil. Ministério da Saúde. Instituto Nacional do Câncer. Controle do câncer de mama: documento de consenso. Rio de Janeiro, 2004 [acesso em 12 jan 2011]. Disponível: http:/www.inca.gov.br/publicacoes/ Consensointegra.pdf

5. Bodstein R. Atenção básica na agenda da saúde. Ciênc. saúde colet. [Internet] 2002;7(3): [acessso em 22 jul 2011]. Disponível: http://www.scielo.br/pdf/csc/ v7n3/13021.pdf

6. Instituto Brasileiro de Geografia e Estatística (IBGE). Censo Demográfico 2010. [acesso em 22 jul 2011]. Disponível em: http://www.ibge.gov.br/home/estatistica/ populacao/censo2010/resultados_dou/ES2010.pdf

7. Associação Brasileira de Empresas de Pesquisa (ABEP). Critério de Classificação Econômica Brasil (CCEB). 2010. [acesso em 22 jul 2011]. Disponível: http://www. abep.org/novo/Content.aspx?ContentID $=302$

8. Schneider IJC, D'orsi E. Sobrevida em cinco anos e fatores prognósticos em mulheres com câncer de mama em Santa Catarina, Brasil. Cad. Saúde Pública. 2009;25(6):1285-96.

9. Leite FMC, Bubach S, Amorim MHC, Castro DS, Primo CC. Mulheres com diagnóstico de câncer de mama em tratamento com tamoxifeno: perfil sociodemográfico e clínico. Rev. bras. cancerol. 2011;57(1):15-21.

10. Crippa CG, Hallal ALC, Dellagiustina AR, Traebert, EE, Gondin G, Pereira C. Perfil clínico e epidemiológico do câncer de mama em mulheres jovens. ACM arq. catarin. med. 2003;32(3):50-8.

11. Brasil. Ministério da Saúde. Instituto Nacional do Câncer. Câncer de mama. [acesso em 24 jun 2011]. Disponível: http://www.saude.gov.br.

12. Trufelli DC, Miranda VC, Santos MBB, Fraile NMP, Pecoroni PG, Gonzaga SFR, et al . Análise do atraso no diagnóstico e tratamento do câncer de mama em um hospital público. Rev. Assoc. Med. Bras. 2008;54(1):72-6.
13. Camargo TC, Souza IEO. Atenção à mulher mastectomizada: discutindo os aspectos ônticos e a dimensão ontológica da atuação da enfermeira no Hospital do Câncer III. Rev. Latino-Am. Enfermagem 2003;11(5):614-21.

14. Brito C, Portela MC, Vasconcellos MTL. Assistência oncológica pelo SUS a mulheres com câncer de mama no Estado do Rio de Janeiro. Rev. Saúde Pública. 2005;39(6):874-81.

15. Lima MG, Koifman S, Scapulatempo IL, Peixoto M, Naomi S, Amaral MC. Fatores de risco para câncer de mama em mulheres indígenas Teréna de área rural, Estado do Mato Grosso do Sul, Brasil. Cad. Saúde Pública. 2001;17:1537-44.

16. Perea SA. Câncer de mama estudo retrospectivo e comparativo do perfil epidemiológico das pacientes em diferentes faixas etárias [dissertação]. São Paulo(SP): Faculdade de Medicina de São José do Rio Preto; 1998.

17. Molina L, Dalben I, De Luca LA. Análise das oportunidades de diagnóstico precoce para as neoplasias malignas de mama. Rev. Assoc. Med. Bras. 2003;49(2):185-90.

18. Monteiro APS, Arraes EPP, Pontes LB, Campos MSS, Ribeiro RT, Gonçalves REB. Auto-exame das mamas: frequência do conhecimento, prática e fatores associados. Rev Bras Ginecol Obstet. 2003;25(3):201-5.

19. Leite FMC, Amorim MHC, Marques GMT, Vilela APM. A estratégia de saúde da família e o rastreamento do câncer de mama. Espac. saude. 2011;12(2):1-9. 